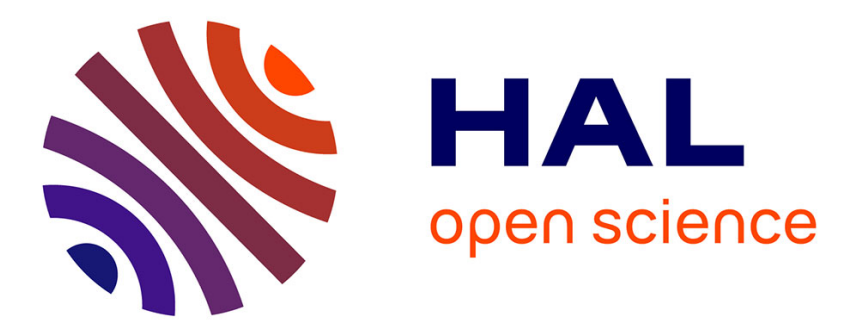

\title{
Robust estimation of the clutter subspace for a Low Rank heterogeneous noise under high Clutter to Noise Ratio assumption
}

\author{
Arnaud Breloy, Guillaume Ginolhac, Frédéric Pascal, Philippe Forster
}

\section{To cite this version:}

Arnaud Breloy, Guillaume Ginolhac, Frédéric Pascal, Philippe Forster. Robust estimation of the clutter subspace for a Low Rank heterogeneous noise under high Clutter to Noise Ratio assumption. 2014 IEEE International Conference on Acoustics, Speech and Signal Processing (ICASSP 2014), May 2014, Florence, Italy. pp.1-5, 10.1109/ICASSP.2014.6853559 . hal-01024200

\section{HAL Id: hal-01024200 \\ https://hal.science/hal-01024200}

Submitted on 15 Jul 2014

HAL is a multi-disciplinary open access archive for the deposit and dissemination of scientific research documents, whether they are published or not. The documents may come from teaching and research institutions in France or abroad, or from public or private research centers.
L'archive ouverte pluridisciplinaire HAL, est destinée au dépôt et à la diffusion de documents scientifiques de niveau recherche, publiés ou non, émanant des établissements d'enseignement et de recherche français ou étrangers, des laboratoires publics ou privés. 


\title{
ROBUST ESTIMATION OF THE CLUTTER SUBSPACE FOR A LOW RANK HETEROGENEOUS NOISE UNDER HIGH CLUTTER TO NOISE RATIO ASSUMPTION
}

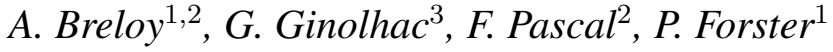 \\ 1 SATIE, ENS Cachan, CNRS, UniverSud, 61, av President Wilson, F-94230 Cachan, France \\ 2 SONDRA, SUPELEC, Plateau du Moulon, 3 rue Joliot-Curie, F-91192 Gif-sur-YvetteCedex, France \\ ${ }^{3}$ LISTIC, Université de Savoie, BP 80439, 74944 ANNECY LE VIEUX Cedex, France
}

\begin{abstract}
In the context of an heterogeneous disturbance with a Low Rank (LR) structure (called clutter), one may use the LR approximation for filtering and detection process. These methods are based on the projector onto the clutter subspace instead of the noise covariance matrix. In such context, adaptive LR schemes have been shown to require less secondary data to reach equivalent performances as classical ones. The main problem is then the estimation of the clutter subspace instead of the noise covariance matrix itself. Maximum Likelihood estimator (MLE) of the clutter subspace has been recently studied for a noise composed of a LR Spherically Invariant Random Vector (SIRV) plus a white Gaussian Noise (WGN). This paper focuses on environments with a high Clutter to Noise Ratio $(C N R)$. An original MLE of the clutter subspace is proposed in this context. A cross-interpretation of this new result and previous ones is provided. Validity and interest - in terms of performance and robustness - of the different approaches are illustrated through simulation results.
\end{abstract}

Index Terms - Covariance Matrix and Projector Estimation, Maximum Likelihood, Low Rank, SIRV, STAP.

\section{INTRODUCTION}

In array processing, many applications require the use of the covariance matrix $(\mathrm{CM})$ of the noise : source localization techniques [1, 2], radar and sonar detection methods [3, 4]. In practice, the $\mathrm{CM}$ of the noise is unknown and has to be estimated from a set of secondary data, i.e. $K$ signal free independent realizations of the noise. The CM estimate is then used to process sub-optimal adaptive methods. Estimating the $\mathrm{CM}$ or its parameters from a given data set is a fundamental issue in signal processing. The CM estimator typically used is the Sample Covariance Matrix (SCM), which is the Maximum Likelihood Estimator (MLE) of the CM in a Gaussian environment. In this case, $K \geqslant 2 M$ (where $M$ is the size of the data) secondary data are needed to ensure good performance of the sub-optimal filtering, i.e. a $3 \mathrm{~dB}$ loss of the output Signal to noise ratio $(S N R)$ compared to optimal filtering [5].
In various applications, the disturbance is not only Gaussian, but a sum of noises with different distributions. In some applications [4], it can be modeled by the sum of a correlated noise, referred to as clutter, plus a white Gaussian noise (WGN). When the correlated disturbance has a low-rank (LR) structure, the corresponding sub-optimal LR-filter is based on the projector on the clutter subspace instead of the CM of the noise $[6,7]$. The advantage of LR method is that estimating the clutter subspace projector requires only $K \geqslant 2 R$ secondary data (where $R$ is the clutter rank, and generally $R \ll M$ ) to reach equivalent performance to the previous scheme [8]. The projector estimate is usually derived from the Singular Value Decomposition (SVD) of an estimate of the CM, classically the SCM. Nevertheless, the SCM is not well adapted for samples that are not Gaussian (presence of outliers, heavy tailed distributions...). Therefore, developing filters/detectors based on the SCM in highly heterogeneous or impulsive clutter environment may lead to poor performance. To describe this kind of disturbance, one of the most general model is the Complex Elliptical Distribution (CED) [9]. Among the general CED class, we will focus on the Spherically Invariant Random Vectors (SIRV) [10]. The SIRV family covers a large panel of well known distributions, notably heavy tailed such as Weibull and K-distribution. Moreover, SIRV presents good agreement to several real clutter data sets $[11,12]$. Eventually, the disturbance will be modeled in this paper as a LR-SIRV clutter plus a WGN, model already used in former works such as $[7,13,14,15]$.

Moreover, an other major issue is the introduction of an outlier into the secondary data. To prevent loss of performances of the estimators due to this corruption, one may derive the projector's estimate from robust estimates of the CM such as Tyler's estimate [16], also known as the Fixed Point Estimator (FPE) [17] in the complex case. However, this estimator require $K \geqslant M$ to be computed, which does not allow to take full advantage of the LR assumption in the cases where $2 R \ll M$. The projector estimate may also be derived from the Normalized SCM (NSCM), which has been shown to be robust [18] but leads to a loss of performance in terms of $S N R$ [15]. The underlying motivation of this work is there- 
fore to provide an estimation method that is both robust and efficient with few secondary data.

This paper proposes to derive the MLE of the clutter subspace projector in this context of LR-SIRV clutter plus a WGN. The problem has recently been addressed in [13], where a solution is provided under two hypothesis : the Probability Density Function (PDF) of the SIRV texture is assumed known, and the LR-SIRV CM is assumed to have identical eigenvalues. [14] presents a "distribution free" version of this result where the texture is treated as an unknown deterministic parameter. In this work, we investigate the high Clutter to Noise Ratio $(C N R)$ scenario, for which the hypothesis of eguals eigenvalues will not be necessary. The MLE of the clutter subspace projector is derived under this assumption and shown to be obtained by a LR version of the FPE. The interest of this result is that it allows to perform robust estimation with a number of secondary data $K<M$. A recent approach to achieve this purpose is to estimate the CM using shrinkage algorithms of the FPE [19, 20, 21]. However the FPE is not an exact MLE of the CM in the described context (LR-SIRV plus WGN). Our approach is different since the LR adaptive method allows to estimate only dominants eigenvectors of the noise CM instead of the CM itself. This method requires less secondary data and will be shown to be more robust to outliers through an application to STAP [4].

The following convention is adopted: italic indicates a scalar quantity, lower case boldface indicates a vector quantity and upper case boldface a matrix. ${ }^{H}$ denotes the transpose conjugate operator and $T$ the transpose operator. $\mathscr{C} \mathscr{N}(\mathbf{a}, \boldsymbol{\Sigma})$ is a complex Gaussian vector of mean a and of covariance matrix $\boldsymbol{\Sigma}$. $E(\tau)$ is the expectation of the random variable $\tau . \mathbf{I}_{M}$ is the $M \times M$ identity matrix. $|\boldsymbol{\Sigma}|$ is the determinant of the matrix $\boldsymbol{\Sigma} . \hat{d}$ is an estimate of the parameter $d .\left\{w_{n}\right\}_{n \in \llbracket 1, N \rrbracket}$ denotes the set of $n$ elements $w_{n}$ with $n \in \llbracket 1, n \rrbracket$ and whose writing will often be contracted into $\left\{w_{n}\right\} . \dagger^{\dagger}$ will denote the rank $R$ pseudo inverse operator: for a given $M \times M$ matrix $\mathbf{M}$, with eigen decomposition $\mathbf{M}=\sum_{r=1}^{M} \lambda_{r} \mathbf{v}_{r} \mathbf{v}_{r}^{H}, \mathbf{M}^{\dagger R}=\sum_{r=1}^{R} 1 / \lambda_{r} \mathbf{v}_{r} \mathbf{v}_{r}^{H}$.

\section{RELATION TO PRIOR WORK}

This paper presents an original MLE of the clutter subspace projector in the context of LR-SIRV clutter plus a WGN. Such an estimator had been derived in [13] (for known SIRV texture PDF) and [14] (for unknown SIRV texture PDF). Both papers use the assumption of identical eigenvalues of the LR-SIRV CM. We relax here this hypothesis using the High CNR assumption, which is more realistic for most applications. Under this assumption, the clutter subspace MLE is shown to be obtained via a LR version of the FPE. The interest of this result is that it allows to perform robust estimation with a number of secondary data $K<M$. A recent approach to achieve this purpose is to estimate the CM using shrinkage algorithms of the FPE [19, 20, 21]. This work presents a different type of shrinkage, using the pseudo-inverse, which is relevant for
LR noises. Another solution to achieve robustness with few data is the use of the NSCM $[18,15]$ but this leads to a loss of performance in terms of $S N R$. All these methods are consequently compared to our approach in terms of performance and robustness through simulations.

\section{MODEL}

We assume that $K$ secondary data are available. The noise is modeled as a LR-SIRV process plus an additive zero-mean complex WGN. A SIRV is a Gaussian random vector with a random power factor called the texture $\tau$. The texture is here considered as an unknown deterministic positive parameter. Therefore, each data $\mathbf{z}_{k} \in \mathbb{C}^{M}, k \in \llbracket 1, K \rrbracket$ can be described, conditioning to $\tau_{k}$, by $\mathbf{z}_{k} \sim \mathscr{C} \mathscr{N}\left(\mathbf{0}, \boldsymbol{\Sigma}_{k}\right)$, with

$$
\boldsymbol{\Sigma}_{k}=\tau_{k} \boldsymbol{\Sigma}_{c}+\sigma^{2} \mathbf{I}_{M},
$$

where $\sigma^{2} \mathbf{I}_{m}$ represents the CM of the WGN, with known $\sigma^{2}$, and $\boldsymbol{\Sigma}_{c}$ the CM of the clutter. $\boldsymbol{\Sigma}_{c}$ is described by its rank $R$, its eigenvalues $c_{r}$ and associated eigenvectors $\mathbf{v}_{r}, r \in \llbracket 1, R \rrbracket$. The clutter rank $R$ is assumed to be known.

$$
\boldsymbol{\Sigma}_{c}=\sum_{r=1}^{R} c_{r} \mathbf{v}_{r} \mathbf{v}_{r}^{H}
$$

Let us now address the problem of the estimation of the cutter subspace projector $\Pi_{c}$ :

$$
\mathbf{\Pi}_{c}=\sum_{r=1}^{R} \mathbf{v}_{r} \mathbf{v}_{r}^{H}
$$

Equation (3) shows that this problem is equivalent to the estimation of a basis of the clutter subspace $\left\{\mathbf{v}_{r}\right\}$. The likelihood of the data set, conditioning to $\left\{\mathbf{v}_{r}\right\}$ and $\left\{\tau_{k}\right\}$, is then:

$$
f\left(\left\{\mathbf{z}_{k}\right\} \mid\left\{\mathbf{v}_{r}\right\},\left\{\tau_{k}\right\}\right)=\prod_{k=1}^{K} \frac{e^{-\mathbf{z}_{k}^{H} \boldsymbol{\Sigma}_{k}^{-1} \mathbf{z}_{k}}}{\pi^{M}\left|\boldsymbol{\Sigma}_{k}\right|}
$$

which leads to the log-likelihood expression:

$$
\ln (f)=-\sum_{k=1}^{K} \mathbf{z}_{k}^{H} \boldsymbol{\Sigma}_{k}^{-1} \mathbf{z}_{k}-\sum_{k=1}^{K} \ln \left(\left|\boldsymbol{\Sigma}_{k}\right|\right)-M K \ln \pi
$$

An approached $\mathrm{MLE}^{1}$ of the clutter subspace basis corresponding to this model is provided in [14]. From now, the $C N R$ will be considered high, which is a realistic assumption for most applications. High $C N R$ means that the WGN is negligible compared to the SIRV noise over the clutter subspace: otherwise stated, for any $r \in \llbracket 1, R \rrbracket, \sigma^{2} \ll \tau_{k} c_{r} \forall k$. Conditioning to $\tau_{k}$, the approached noise CM for a sample $\mathbf{z}_{k}$ is then:

$$
\boldsymbol{\Sigma}_{k} \approx \tau_{k} \sum_{r=1}^{R} c_{r} \mathbf{v}_{r} \mathbf{v}_{r}^{H}+\sigma^{2} \sum_{r=R+1}^{M} \mathbf{v}_{r} \mathbf{v}_{r}^{H}
$$

\footnotetext{
${ }^{1}$ based on the approximation of equals $c_{r}$ 's.
} 
where $\left\{\mathbf{v}_{r}\right\}_{r>R}$ is the completion of the basis $\left\{\mathbf{v}_{r}\right\}_{r \in \llbracket 1, R \rrbracket}$. Combining equations (4) and (6), one can derive the loglikelihood of the data set in high $C N R$ scenario. Conditioning to $\left\{\mathbf{v}_{r}\right\}$ and $\left\{\tau_{k}\right\}$ its expression is given by:

$$
\begin{array}{r}
\ln f=-\sum_{k=1}^{K} \sum_{r=1}^{R} \frac{1}{\tau_{k} c_{r}} \mathbf{z}_{k}^{H} \mathbf{v}_{r} \mathbf{v}_{r}^{H} \mathbf{z}_{k} \\
-\sum_{k=1}^{K} \sum_{r=R+1}^{M} \frac{1}{\sigma^{2}} \mathbf{z}_{k}^{H} \mathbf{v}_{r} \mathbf{v}_{r}^{H} \mathbf{z}_{k}-R \sum_{k=1}^{K} \ln \left(\tau_{k}\right) \\
-K \sum_{r=1}^{R} \ln \left(c_{r}\right)-K(M-R) \ln \left(\sigma^{2}\right)-M K \ln \pi
\end{array}
$$

\section{MLE OF $\Pi_{C}$ AND DISCUSSION}

Proposition 4.1 The MLE of the clutter subspace basis $\left\{\hat{\mathbf{v}}_{r}\right\}$ is given by the $R$ greatest eigenvectors of the matrix $\hat{\boldsymbol{\Sigma}}_{L R-F P}$ that satisfies:

$$
\hat{\boldsymbol{\Sigma}}_{L R-F P}=\sum_{k=1}^{K} \frac{\mathbf{z}_{k} \mathbf{z}_{k}^{H}}{\mathbf{z}_{k}^{H} \hat{\mathbf{\Sigma}}_{L R-F P}^{\dagger R} \mathbf{z}_{k}}
$$

where $\hat{\boldsymbol{\Sigma}}_{L R-F P}^{\dagger_{R}}$ is the rank $R$ pseudo inverse of the matrix $\boldsymbol{\Sigma}_{L R-F P}$ :

$$
\hat{\boldsymbol{\Sigma}}_{L R-F P}^{\dagger R}=\sum_{r=1}^{R} \frac{1}{\hat{c}_{r}} \hat{\mathbf{v}}_{r} \hat{\mathbf{v}}_{r}^{H}
$$

Proof 4.1 The textures $\left\{\tau_{k}\right\}$ are unknown deterministic parameters. The first step of the proof is deriving their MLE expression from the log-likelihood. (7) is differentiated with respect to (w.r.t.) $\tau_{k}$ and canceled for

$$
\hat{\tau}_{k}=\frac{1}{R} \sum_{r=1}^{R} \frac{1}{c_{r}} \mathbf{z}_{k}^{H} \mathbf{v}_{r} \mathbf{v}_{r}^{H} \mathbf{z}_{k}=\frac{1}{R} \mathbf{z}_{k}^{H} \boldsymbol{\Sigma}_{c}^{\dagger R} \mathbf{z}_{k}
$$

The parameters $\left\{\tau_{k}\right\}$ are substituted by their MLE expression in (7) to obtain the generalized log-likelihood:

$$
\begin{array}{r}
\ln (f)=-K R-R \sum_{k=1}^{K} \ln \left(\frac{1}{R} \mathbf{z}_{k}^{H} \mathbf{\Sigma}_{c}^{\dagger} \mathbf{z}_{k}\right) \\
-\sum_{k=1}^{K} \sum_{r=R+1}^{M} \frac{1}{\sigma^{2}} \mathbf{z}_{k}^{H} \mathbf{v}_{r} \mathbf{v}_{r}^{H} \mathbf{z}_{k}-K \sum_{r=1}^{R} \ln \left(c_{r}\right) \\
-K(M-R) \ln \left(\sigma^{2}\right)-M K \ln \pi
\end{array}
$$

The vector set $\left\{\mathbf{v}_{r}\right\}$ must form a basis of the clutter subspace estimate. Thus, the maximization of $\ln \hat{f}$ with respect to the $\mathbf{v}_{r}$ 's must be done under a normalization constraint. Nevertheless, imposing an orthogonality constraint is not necessary since the solution will appear as eigenvectors of an unique matrix and therefore inherently orthogonal to each other. Removing terms that are not depending on $\mathbf{v}_{r}$ 's, the functional $g$ to maximize w.r.t the $\mathbf{v}_{r}$ 's is:

$$
g=-R \sum_{k=1}^{K} \ln \left(\frac{1}{R} \mathbf{z}_{k}^{H} \mathbf{\Sigma}_{c}^{\dagger_{R}} \mathbf{z}_{k}\right)+\sum_{r=1}^{R} \lambda_{r}\left(\mathbf{v}_{r}^{H} \mathbf{v}_{r}-1\right)
$$

where $\lambda_{r}, r \in \llbracket 1, R \rrbracket$ are Lagrange multipliers associated to the normalization constraint. $g$ is differentiated, w.r.t. $\mathbf{v}_{j}^{H}$ for a specific $j \in \llbracket 1, R \rrbracket$, and canceled for:

$$
\frac{\partial g}{\partial \mathbf{v}_{j}^{H}}=0 \Leftrightarrow R \sum_{k=1}^{K} \frac{\mathbf{z}_{k} \mathbf{z}_{k}^{H}}{\mathbf{z}_{k}^{H} \sum_{r=1}^{R} \frac{1}{c_{r}} \mathbf{v}_{j} \mathbf{v}_{j}^{H} \mathbf{z}_{k}} \mathbf{v}_{j}=\frac{\lambda_{j}}{c_{j}} \mathbf{v}_{j}
$$

Thus, the ML basis of the clutter subspace is defined as eigenvectors of the matrix $\hat{\boldsymbol{\Sigma}}_{L R-F P}$ that satisfies the fixed point equation (8). This result considered with a maximization objective of $f$ concludes the proof of proposition 4.1.

The introduced estimator is defined as the fixed point of an implicit function. We propose the following heuristic:

$$
\boldsymbol{\Sigma}_{(n+1)}=\sum_{k=1}^{K} \frac{\mathbf{z}_{k} \mathbf{z}_{k}^{H}}{\mathbf{z}_{k}^{H} \boldsymbol{\Sigma}_{(n)}^{\dagger R} \mathbf{z}_{k}},
$$

to compute $\hat{\boldsymbol{\Sigma}}_{L R-F P}$, which corresponds to the FPE algorithm, but wit a rank $R$ pseudo inverse. Convergence and uniqueness of the solution is not yet proven and should be the topic of further coming work.

The MLE of the cluter subspace presented in this paper and in [14] are both defined as the $R$ dominant eigenvectors of a matrix. It is important to notice that this matrices are just intermediary results and not MLE of the noise CM itself. These matrices correspond to SCMs of the data scaled by a factor that is depending on the estimated texture. The interpretation of these two factors denotes a rather different estimation strategy. In the "classical" case [13, 14], the samples $\mathbf{z}_{k} \mathbf{z}_{k}^{H}$ are scaled by a factor $\frac{\hat{\tau}_{k}}{\hat{\tau}_{k}+\sigma^{2}} \in[0,1]$ : the Clutter to Overall Noise Ratio $(C O N R)$ estimate. With these scaling factors, realizations that contain more power in the subspace of interest are given more significance in the estimation process, which may be useful if the clutter subspace is not well represented over the data set (for example with a low $C N R$ or for very impulsive SIRV's). In the high $C N R$ case (Proposition 4.1) the samples $\mathbf{z}_{k} \mathbf{z}_{k}^{H}$ are scaled by the inverse of the estimated texture $\frac{1}{\hat{\tau}_{k}}$. The matrix $\boldsymbol{\Sigma}_{L R}$ corresponds to the FPE with a rank shrinkage. A robust estimation strategy is therefore applied over the subspace of interest. This LR version of the FPE may be of interest since it proposes a robust estimation method that does not need regularization algorithms such as in [20,21] as long as $K>R$ (instead of $K>M$ in the classical FPE algorithm).

\section{APPLICATION TO STAP}

First of all, let us denote by $\hat{\boldsymbol{\Pi}}_{S C M}, \hat{\boldsymbol{\Pi}}_{N S C M}, \hat{\boldsymbol{\Pi}}_{S-F P E}$, respectively the clutter subspace estimates derived from an 


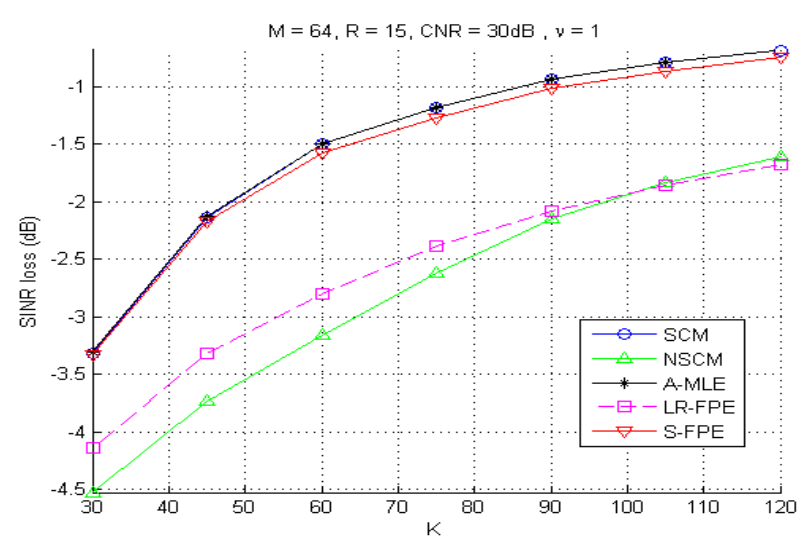

Fig. 1. SINR-Loss versus $K$, for the following STAP configuration: $Q=$ $8, P=8, M=P Q=64$. Center frequency $f_{0}=450 \mathrm{MHz}$, bandwidth $B=4 \mathrm{MHz}$. Radar velocity $v=100 \mathrm{~m} / \mathrm{s}$. Inter-element spacing $d=$ $\frac{c}{2 f_{0}}$ with $c$ the celerity of light. Pulse repetition frequency $f_{r}=600 \mathrm{~Hz}$. Clutter rank $R=15$ computed from Brennan rule [22]. The texture PDF is a Gamma law of shape parameter $\nu=1$ and scale parameter $\frac{1}{\nu}$. $C N R=$ $30 \mathrm{~dB}$.

SVD of the SCM, the NSCM, and the diagonnaly loaded FPE $[19,20]$.

STAP is applied to airborne radar in order to detect moving targets. Typically, the radar receiver consists in an array of $Q$ antenna elements processing $P$ pulses in a coherent processing interval $(M=P Q)$. In this framework, we assume that the received signal $\mathbf{z}=\mathbf{d}+\mathbf{n}$ is a complex known signal $\mathbf{d}$ corrupted by an additive disturbance $\mathbf{n}$ which follows the general noise model described in section 3. With a LR clutter, it is well known that a classical sub-optimal filter is $[6,8]$ :

$$
\hat{\mathbf{w}}_{l r}=\hat{\boldsymbol{\Pi}}_{c}^{\perp} \mathbf{d}=\left(\mathbf{I}_{m}-\hat{\mathbf{\Pi}}_{c}\right) \mathbf{d}
$$

Of course, the performance of the LR filters will directly rely on the accuracy of the estimation of $\boldsymbol{\Pi}_{c}$, which will illustrate performance of the clutter subspace estimators. The criterion used is the mean SINR-Loss [4]: the ratio between the $S I N R_{\text {out }}$, computed for $\hat{\mathbf{w}}_{l r}$, and $S I N R_{\max }$ computed for the optimal filter $\mathbf{w}=\boldsymbol{\Sigma}^{-1} \mathbf{d}$, with $\boldsymbol{\Sigma}=\sigma^{2} \mathbf{I}+E(\tau) \boldsymbol{\Sigma}_{c}$.

Figure 1 presents the mean SINR-Loss versus the number of secondary data $K: \hat{\boldsymbol{\Pi}}_{A-M L E}, \hat{\boldsymbol{\Pi}}_{S C M}$ and $\hat{\boldsymbol{\Pi}}_{S-F P E}$ reach a slightly better SINR-Loss than $\hat{\boldsymbol{\Pi}}_{L R-F P E}$, and $\hat{\boldsymbol{\Pi}}_{N S C M}$ is below. In Figure 2 the steering vector of the target is inserted into the secondary data. The presence of this outlier in the estimation data set deteriorates the performance of the adaptive filter. An estimator is more robust to outliers if its drop in mean SINR-Loss comes for a higher Outlier to Noise Ratio $(O N R)$. In that case, $\hat{\mathbf{\Pi}}_{L R-F P E}$ appears to be more robust than others estimators. Figure 3 shows the output of adaptive filters build from different estimators for two scenarios : with and without an outlier in the training set. This illustrates the interest of the proposed approach : $\hat{\boldsymbol{\Pi}}_{L R-F P E}$ does not causes a great loss in performance with uncorrupted

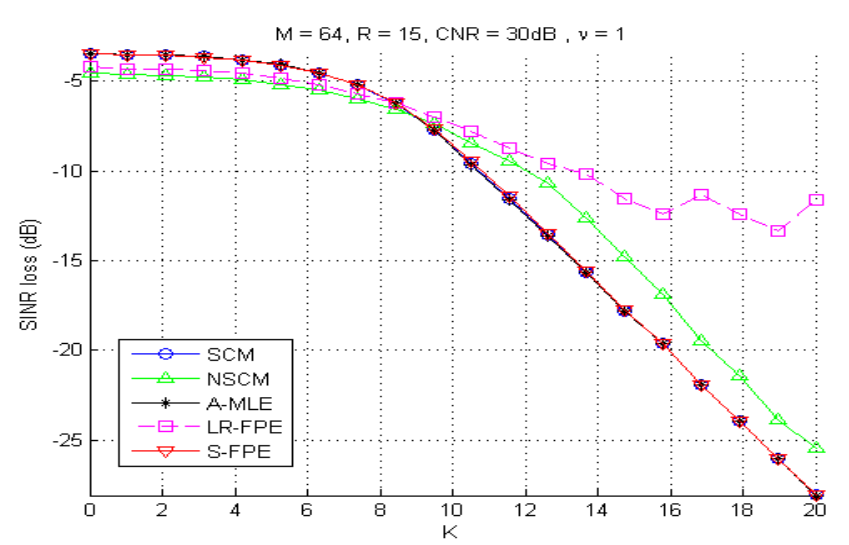

Fig. 2. SINR-Loss versus $O N R$, for the same configuration, $K=2 R$.
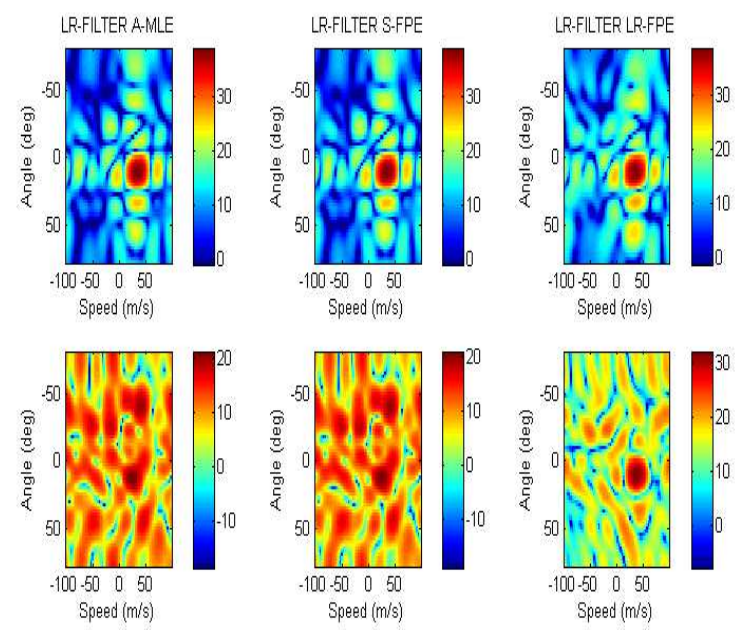

Fig. 3. Output of the adaptive filters build from A-MLE (left) S-FPE (middle) and LR-FPE (right). $K=2 R$, uncorrupted training set (top) and corrupted training set (bottom) with $O N R=15 \mathrm{~dB}$. Target at $10^{\circ}$ and $V_{t}=35 \mathrm{~m} / \mathrm{s}$ with $S N R=20 \mathrm{~dB}$

samples but presents attractive robust properties since it provides a better interference rejection when training samples are corrupted.

\section{CONCLUSION}

This paper has presented a new MLE of the clutter subspace basis in the context of a LR-SIRV plus WGN under the high $C N R$ assumption. This MLE has been shown to be the dominant eigenvectors of a LR version of the FPE [16][17]. This result allows to perform robust estimation of the clutter subspace with few data $(K>R$ instead of $K>M$ for the FPE) in LR heterogeneous environment. Simulation results show that this estimator is valid and seems to ensure a good compromise between performance (in terms SINR-Loss) and robustness to outliers for small training sets $(K \simeq 2 R)$. 


\section{REFERENCES}

[1] R. O. Schmidt, "Multiple emitter location and signal parameter estimation," IEEE Trans.-ASSP, vol. 34, no. 3, pp. 276-280, March 1986.

[2] R. Roy and T. Kailath, "ESPRIT-Estimation of signal parameters via rotational invariant techniques," IEEE Trans.-ASSP, vol. 37, no. 7, pp. 984-995, July 1989.

[3] S. M. Kay, Fundamentals of Statistical Signal Processing - Detection Theory, vol. 2, Prentice-Hall PTR, 1998.

[4] J. Ward, "Space-time adaptive processing for airborne radar," Tech. Rep., Lincoln Lab., MIT, Lexington, Mass., USA, December 1994.

[5] I.S. Reed, J.D. Mallett, and L.E. Brennan, "Rapid convergence rate in adaptive arrays," IEEE Trans. on Aero. and Elec. Syst., vol. AES-10, no. 6, pp. 853 - 863, November 1974.

[6] I. Kirsteins and D. Tufts, "Adaptive detection using a low rank approximation to a data matrix," IEEE Trans. on Aero. and Elec. Syst., vol. 30, pp. 55 - 67, 1994.

[7] M. Rangaswamy, F.C. Lin, and K.R. Gerlach, "Robust adaptive signal processing methods for heterogeneous radar clutter scenarios," Signal Processing, vol. 84, pp. $1653-1665,2004$.

[8] A. Haimovich, "Asymptotic distribution of the conditional signal-to-noise ratio in an eigenanalysis-based adaptive array," IEEE Trans. on Aero. and Elec. Syst., vol. 33, pp. 988 - 997, 1997.

[9] E. Ollila, J. Eriksson, and V. Koivunen, "Complex elliptically symmetric random variables-generation, characterization, and circularity tests," Signal Processing, IEEE Transactions on, vol. 59, no. 1, pp. 58-69, 2011.

[10] K. Yao, "A representation theorem and its applications to spherically invariant random processes," IEE Trans. on Inf. Th., vol. 19, no. 5, pp. 600 - 608, September 1973.

[11] J.B. Billingsley, "Ground clutter measurements for surface-sited radar," Tech. Rep. 780, MIT, February 1993.

[12] M. Rangaswamy, D.D. Weiner, and A. Ozturk, "Nongaussian vector identification using spherically invariant random processes," IEEE Trans.-AES, vol. 29, no. 1, pp. 111-124, January 1993.

[13] R.S. Raghavan, "Statistical interpretation of a data adaptive clutter subspace estimation algorithm," IEEE Trans. on Aero. and Elec. Syst., vol. 48, no. 2, pp. 1370 - 1384, 2012.
[14] A. Breloy, L. Le Magoarou, G. Ginolhac, F. Pascal, and P. Forster, "Maximum likelihood estimation of clutter subspace in non homogeneous noise context," in Proceedings of EUSIPCO, Marrakech, Morocco, September 2013.

[15] G. Ginolhac, P. Forster, F. Pascal, and J.-P. Ovarlez, "Performance of two low-rank stap filters in a heterogeneous noise," Signal Processing, IEEE Transactions on, vol. 61, no. 1, pp. 57-61, 2013.

[16] D. Tyler, "A distribution-free m-estimator of multivariate scatter," The Annals of Statistics, vol. 15, no. 1, pp. 234-251, 1987.

[17] F. Pascal, Y. Chitour, J.P. Ovarlez, P. Forster, and P. Larzabal, "Existence and characterization of the covariance matrix maximum likelihood estimate in spherically invariant random processes," IEEE Trans on Sig. Proc., vol. 56, no. 1, pp. 34 - 48, January 2008.

[18] G. Ginolhac, P. Forster, J.P. Ovarlez, and F. Pascal, "Spatio-temporal adaptive detector in nonhomogeneous and low-rank clutter," in Proceedings of ICASSP, Taipei, Taïwan, april 2009.

[19] Y. Abramovich and O. Besson, "Regularized covariance matrix estimation in complex elliptically symmetric distributions using the expected likelihood approach - part 1: The over-sampled case," Signal Processing, IEEE Transactions on, vol. PP, no. 99, pp. 1-1, 2013.

[20] O. Besson and Y. Abramovich, "Regularized covariance matrix estimation in complex elliptically symmetric distributions using the expected likelihood approach - part 2: The under-sampled case," Signal Processing, IEEE Transactions on, vol. PP, no. 99, pp. 1-1, 2013.

[21] A. Wiesel, "Unified framework to regularized covariance estimation in scaled gaussian models," Signal Processing, IEEE Transactions on, vol. 60, no. 1, pp. 29-38, 2012.

[22] L. E. Brennan and F.M. Staudaher, "Subclutter visibility demonstration," Tech. Rep., RL-TR-92-21, Adaptive Sensors Incorporated, March 1992. 